Pacific Journal of Mathematic 


\title{
TOTALLY POSITIVE DIFFERENTIAL SYSTEMS
}

\author{
BINYAMIN SCHWARZ
}

Totally positive (TP), and strictly totally positive (STP) differential systems are defined. These real, first order, linear systems are characterized by the form of their coefficient matrices, and by the decrease of the number of sign changes of their solution vectors as functions of the independent variable. A bound is given for the combined number of zeros of the first and last components of any particular solution vector of STP system and a similar result is obtained for TP systems. Examples show that no such bounds exist for the number of zeros of any other component.

In this paper we consider real differential systems of the form

$$
y^{\prime}(t)=A(t) y(t) \text {. }
$$

Here the solutions $y(t)$ are real column vectors $y(t)=\left(y_{1}(t), \cdots, y_{n}(t)\right)$ and $A(t)$ is a given $n \times n$ matrix $\left(a_{i j}(t)\right)_{1}^{n}$ whose elements $a_{i j}(t)$ are real functions which are continuous in the open interval $(a, b),-\infty \leqq$ $a<b \leqq \infty$. Together with the vector differential equation (1.1) we consider also the corresponding matrix differential equation

$$
Y^{\prime}(t)=A(t) Y(t),
$$

where $Y(t)=\left(y_{i j}(t)\right)_{1}^{n}$. Let $Y(t)$ be any solution of (1.2); for each integer $p, 1 \leqq p \leqq n$, we denote the $p$ th compound of $Y(t)$ by $C_{p}(Y(t))$. In $\S 2$ we construct for each $p, 1 \leqq p \leqq n$, a $\left(\begin{array}{l}n \\ p\end{array}\right) \times\left(\begin{array}{l}n \\ p\end{array}\right)$ matrix $B^{(p)}(t)$, such that

$$
\left[C_{p}(Y(t))\right]^{\prime}=B^{(p)}(t) C_{p}(Y(t)) .
$$

$\left(B^{(1)}(t)=A(t)\right.$.) The elements of $B^{(p)}(t)$ are easily expressed by the given $n^{2}$ elements $a_{i j}(t)$ of $A(t)$ (Theorem 1). Special cases of these compound systems were previously considered: Mikusiński [6] considered the differential system satisfied by the $2 \times 2$ Wronskians of the solutions of the equation $u^{(n)}(t)+p(t) u(t)=0$ and Nehari [7] considered all compound systems (1.3) in the case where (1.1) is equivalent to an $n$th order linear differential equation. We remark that for $p=n-1(1.3)$ is closely related to the system adjoint to (1.2); and for $p=n$ (1.3) reduces to Liouville's equation

$$
\Delta(t)^{\prime}=\left(\sum_{i=1}^{n} a_{i i}(t)\right) \Delta(t),
$$

where $\Delta(t)=C_{n}(Y(t))$ is the determinant of $Y(t)$. We state an im- 
mediate consequence of Theorem 1, showing a characteristic difference between the elements $a_{i j}(t)$ with $|i-j|=1$ and the other off-diagonal elements of $A(t)$, as a corollary.

A real $n \times n$ matrix is totally positive (TP) is all its minors are nonnegative, and the matrix is strictly totally positive (STP) if all its minors are positive. For each $r, a<r<b$, we denote the fundamental solution $Y(t)$ of (1.2) satisfying

$$
Y(r)=I,
$$

$\left(I=\left(\delta_{i j}\right)_{1}^{n}\right)$, by $Y(t)=Y(t, r)$. We call the system (1.2), and the corresponding system (1.1), totally positive (TP) in $(a, b)$ if for each pair $(r, t), a<r \leqq t<b, Y(t, r)$ is TP. If for each pair $(r, t), a<$ $r<t<b, Y(t, r)$ is STP then the systems (1.2) and (1.1) are called strictly totally positive (STP) in $(a, b)$. In $\S 3$ we characterize these systems by the form of the matrix $A(t)=\left(a_{i j}(t)\right)_{1}^{n}$. The system (1.2) is TP in $(a, b)$ if and only if $A(t)$ is a (variable) Jacobi matrix (i.e., $a_{i j}(t) \equiv 0$ for $|i-j| \geqq 2$ ) with nonnegative off-diagonal elements (i.e., $\left.a_{i, i+1}(t) \geqq 0, a_{i+1, i}(t) \geqq 0, i=1, \cdots, n-1\right)$. This result (Theorem 2) was first proved by Loewner [5]. Our proof (based on Corollary 1) is quite elementary and leads also to the following modification of Loewner's result: The system (1.2) is STP in $(a, b)$ if and only if $A(t)$ satisfies the above conditions and none of the functions $a_{i, i+1}(t)$ and $a_{i+1, i}(t)$ vanishes identically in any interval contained in $(a, b)$ (Theorem 3).

In $\S 4$ we consider vector solutions $y(t)$ of a STP system. The system (1.1) is shown to be STP in $(a, b)$ if and only if $S^{+}(y(s)) \leqq S^{-}(y(r))$ holds for all nontrivial solutions $y(t)$ and all pairs $(r, s), a<r<s<b$, (Theorem 4). This result on the number of sign changes, following from the variation-diminishing properties of STP matrices, leads now to results on the number of zeros of the components $y_{1}(t)$ and $y_{n}(t)$ of any given vector solution $y(t)$ of (1.1). The combined number of zeros of these two extreme components cannot exceed $n-1$ (Theorem 5). No such restriction exists for the interior components $y_{2}(t), \cdots, y_{n-1}(t)$. We illustrate this dissimilarity between the extreme and the interior components by examples in the last section $(\S 6)$. In $\S 5$ we consider vector solutions of TP systems and the results are now weakened versions of the corresponding results for STP systems. We rely strongly on the recent book by Karlin [4], but we give all necessary definitions in order to keep this paper reasonably selfcontained.

2. The compound differential systems. For given integers $n$ and $p, 1 \leqq p \leqq n$, we consider the $p$-tuples of increasing integers 


$$
1 \leqq \begin{aligned}
& i_{1}<i_{2}<\cdots<i_{p} \\
& j_{1}<j_{2}<\cdots<j_{p}
\end{aligned} \leqq n,
$$

and we arrange these $N=\left(\begin{array}{l}n \\ p\end{array}\right) p$-tuples in lexicographic order. We denote these $p$-tuples of indices also by

$$
\alpha=\left(i_{1}, i_{2}, \cdots, i_{p}\right), \quad \beta=\left(j_{1}, \cdots, j_{p}\right) .
$$

For a $n \times n$ matrix $Y=\left(y_{i j}\right)_{1}^{n}$, we denote the minor, determined by these rows and columns, by

$$
Y\left(\begin{array}{l}
i_{1}, i_{2}, \cdots, i_{p} \\
j_{1}, j_{2}, \cdots, j_{p}
\end{array}\right)
$$

The $p$ th compound $C_{p}(Y)$ of $Y$ is the $N \times N$ matrix having these minors (in lexicographic order) as elements. The elements of the $N \times N$ matrix $B^{(p)}$ are denoted by $b_{\alpha \beta}=b\left(i_{1}, \cdots, i_{p} \mid j_{1}, \cdots, j_{p}\right)$. In the following the matrices $A$ and $B^{(p)}$ will be continuous functions of $t, Y$ and $C_{p}(Y)$ will therefore be continuously differentiable functions of $t$. Using this notation we obtain the following relation between the given system (1.2) and its compound systems (1.3).

Theorem 1. Let $Y(t)=\left(y_{i j}(t)\right)_{1}^{n}, a<t<b,-\infty \leqq a<b \leqq \infty$, be any solution of the differential system

$$
Y^{\prime}(t)=A(t) Y(t),
$$

where $A(t)=\left(a_{i j}(t)\right)_{1}^{n}$ and the $n^{2}$ real functions $a_{i j}(t)$ are continuous in $(a, b)$. The $p$ th compound $C_{p}(Y(t))$ of $Y(t), 1 \leqq p \leqq n$, satisfies in $(a, b)$ the equation

$$
\left[C_{p}(Y(t))\right]^{\prime}=B^{(p)}(t) C_{p}(Y(t))
$$

The matrix $B^{(p)}(t)=\left(b_{\alpha \beta}(t)\right)_{1}^{N}, N=\left(\begin{array}{l}n \\ p\end{array}\right)$, is given by

$$
b_{\alpha \beta}(t)=b\left(i_{1}, \cdots, i_{p} \mid j_{1}, \cdots, j_{p}\right)=\left\{\begin{array}{l}
0 \text { if at most } p-2 \text { of the indices } \\
i \text { of } \alpha \text { coincide with indices } j \\
\text { of } \beta ; \\
(-1)^{\ell+m} a_{i}{ }^{j_{m}} \text { if exactly } p-1 \text { of } \\
\text { the indices of } \alpha \text { coincide with } \\
\text { indices of } \beta, \text { but } i_{\ell} \neq j_{m}, 1 \leqq \\
\ell, m \leqq p ; \\
\sum_{\ell=1}^{p} a_{i} \iota^{j} \ell \text { if } i_{\ell}=j_{\ell}, \iota=1, \cdots, p .
\end{array}\right.
$$

Proof. We choose two $p$-tuples of increasing indices $\alpha=\left(i_{1}, \cdots, i_{p}\right)$, 
$\gamma=\left(k_{1}, \cdots, k_{p}\right)$ with

$$
1 \leqq \begin{aligned}
& i_{1}<i_{2}<\cdots<i_{p} \\
& k_{1}<k_{2}<\cdots<k_{p}
\end{aligned} \leqq n
$$

and consider the minor $Y\left(\begin{array}{l}i_{1}, \cdots, i_{p} \\ k_{1}, \cdots, k_{p}\end{array}\right)$. Differentiating this minor by rows and using (1.2) we obtain

$$
\begin{aligned}
Y\left(\begin{array}{c}
i_{1}, \cdots, i_{p} \\
k_{1}, \cdots, k_{p}
\end{array}\right)^{\prime}= & \left|\begin{array}{ccc}
\sum_{\nu=1}^{n} a_{i_{1} \nu} y_{\nu k_{1}} & \cdots & \sum_{\nu=1}^{n} a_{i_{1} \nu} y_{\nu k_{p}} \\
\ldots & \cdots & \cdots \\
y_{i_{p} k_{1}} & \cdots & y_{i_{p} k_{p}}
\end{array}\right|+\cdots \\
& +\left|\begin{array}{ccc}
y_{i_{1} k_{1}} & \cdots & y_{i_{1} k_{p}} \\
\cdots & \cdots & \cdots \\
\sum_{\nu=1}^{n} a_{i_{p} \nu} y_{\nu k_{1}} & \cdots & \sum_{\nu=1}^{n} a_{i_{p^{\nu}}} y_{\nu k_{p}}
\end{array}\right|
\end{aligned}
$$

We rewrite this as

$$
\begin{aligned}
Y\left(\begin{array}{c}
i_{1}, \cdots, i_{p} \\
k_{1}, \cdots, k_{p}
\end{array}\right)^{\prime}= & \sum_{\nu=1}^{n} a_{i_{1^{\nu}}} Y\left(\begin{array}{c}
\nu, i_{2}, \cdots, i_{p} \\
k_{1}, k_{2}, \cdots, k_{p}
\end{array}\right) \\
& +\sum_{\nu=1}^{n} a_{i_{2^{\nu}}} Y\left(\begin{array}{c}
i_{1}, \nu, i_{3}, \cdots, i_{p} \\
k_{1}, k_{2}, k_{3}, \cdots, k_{p}
\end{array}\right)+\cdots \\
& +\sum_{\nu=1}^{n} a_{i_{p^{\nu}}} Y\left(\begin{array}{c}
i_{1}, \cdots, i_{p-1}, \nu \\
k_{1}, \cdots, k_{p-1}, k_{p}
\end{array}\right) .
\end{aligned}
$$

The row indices on the r.h.s are, in general, not in increasing order and the $p n$ determinants appearing there are hence, in general, not minors of $Y$. But each of these determinants either vanishes or is equal to a minor of $Y$ or is equal to $(-1)$ times a minor. We thus can write (2.2) in the form

$$
\begin{aligned}
Y\left(\begin{array}{l}
i_{1}, \cdots, i_{p} \\
k_{1}, \cdots, k_{p}
\end{array}\right)^{\prime} & =\sum_{\beta} b_{\alpha \beta} Y\left(\begin{array}{l}
j_{1}, \cdots, j_{p} \\
k_{1}, \cdots, k_{p}
\end{array}\right) \\
& =\sum_{1 \leqq j_{1}<\cdots<j_{p} \leqq n} b\left(i_{1}, \cdots, i_{p} \mid j_{1}, \cdots, j_{p}\right) Y\left(\begin{array}{l}
j_{1}, \cdots, j_{p} \\
k_{1}, \cdots, k_{p}
\end{array}\right) .
\end{aligned}
$$

To obtain (2.1) we compare (2.2) and (2.3). We first note that on the r.h.s. of (2.2) appear only $p$-tuples of row indices for which at least $p-1$ of the indices belong to the $p$-tuple $\alpha=\left(i_{1}, \cdots, i_{p}\right)$. This gives the first part of (2.1). Secondly, if $\nu$ does not belong to $\alpha$, then the $p$-tuples

$$
\left(\nu, i_{2}, \cdots, i_{p}\right),\left(i_{1}, \nu, i_{3}, \cdots, i_{p}\right), \cdots,\left(i_{1}, \cdots, i_{p-1}, \nu\right),
$$

appearing as row indices on the r.h.s. of (2.2), have to be rearranged 
by putting the index $\nu=j_{m}$ in its proper place in order to obtain an increasing $p$-tuple which in $(2.3)$ is denoted by $\beta=\left(j_{1}, \cdots, j_{p}\right)$. For the $p$-tuples corresponding to the first sum on the r.h.s. of (2.2) this may be achieved by $m+1$ transpositions, for those of the second sum by $m+2$ transpositions and, in general, for those of the $\ell$ th sum by $m+\ell$ transpositions. This implies the second part of (2.1). Finally, if we choose $\nu=i_{1}$ in the first sum on the r.h.s. of (2.2), $\nu=i_{2}$ in the second sum and so on, we obtain the last part of (2.1) and we have thus proved Theorem 1.

We illustrate this result by expressing the elements $b_{\alpha \beta}$ of $B^{(p)}$ in terms of the elements $a_{i j}$ in the simplest cases: $n=3, p=2, n=4$, $p=2$ and $n=4, p=3$.

\begin{tabular}{|c|c|c|c|c|}
\hline$i_{1}$ & $i_{2}$ & $\begin{array}{ll}j_{1} & j_{2} \\
1 & 2\end{array}$ & $\begin{array}{ll}j_{1} & j_{2} \\
1 & 3\end{array}$ & $\begin{array}{ll}j_{1} & j_{2} \\
2 & 3\end{array}$ \\
\hline 1 & 2 & $a_{11}+a_{22}$ & $a_{23}$ & $-a_{13}$ \\
\hline 1 & 3 & $a_{32}$ & $a_{11}+a_{33}$ & $a_{12}$ \\
\hline 2 & 3 & $-a_{31}$ & $a_{21}$ & $a_{22}+a_{33}$ \\
\hline
\end{tabular}

\begin{tabular}{|c|c|c|c|c|c|c|c|}
\hline$i_{1}$ & $i_{2}$ & $\begin{array}{ll}j_{1} & j_{2} \\
1 & 2\end{array}$ & $\begin{array}{ll}j_{1} & j_{2} \\
1 & 3\end{array}$ & $\begin{array}{ll}j_{1} & j_{2} \\
1 & 4\end{array}$ & $\begin{array}{ll}j_{1} & j_{2} \\
2 & 3\end{array}$ & $\begin{array}{ll}j_{1} & j_{2} \\
2 & 4\end{array}$ & $\begin{array}{ll}j_{1} & j_{2} \\
3 & 4\end{array}$ \\
\hline 1 & 2 & $a_{11}+a_{22}$ & $a_{23}$ & $a_{24}$ & $-a_{13}$ & $-a_{14}$ & 0 \\
\hline 1 & 3 & $a_{32}$ & $a_{11}+a_{33}$ & $a_{34}$ & $a_{12}$ & 0 & $-a_{14}$ \\
\hline 1 & 4 & $a_{42}$ & $a_{43}$ & $a_{11}+a_{44}$ & 0 & $a_{12}$ & $a_{13}$ \\
\hline 2 & 3 & $-a_{31}$ & $a_{21}$ & 0 & $a_{22}+a_{33}$ & $a_{34}$ & $-a_{24}$ \\
\hline 2 & 4 & $-a_{41}$ & 0 & $a_{21}$ & $a_{43}$ & $a_{22}+a_{44}$ & $a_{23}$ \\
\hline 3 & 4 & 0 & $-a_{41}$ & $a_{31}$ & $-a_{42}$ & $a_{32}$ & $a_{33}+a_{44}$ \\
\hline
\end{tabular}

$n=4, p=2$

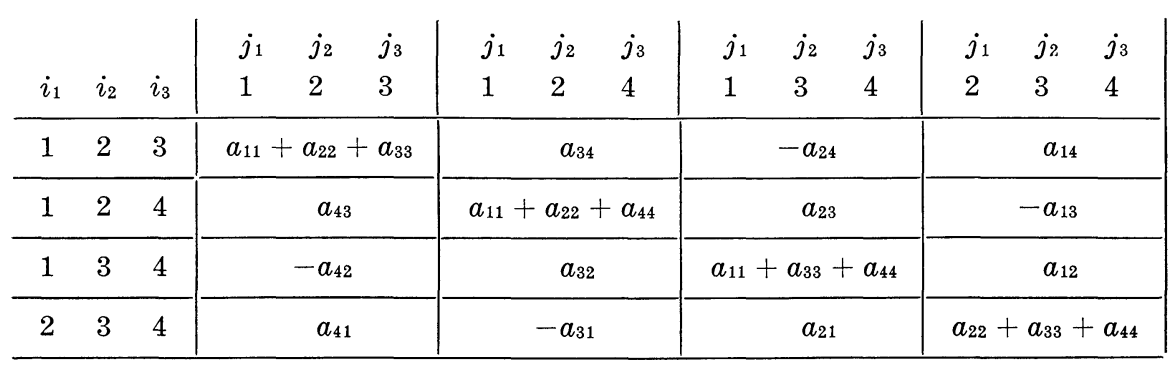


We remark that each diagonal element $a_{i i}$ appears as a summand in $\left(\begin{array}{l}n-1 \\ p-1\end{array}\right)$ diagonal elements of $B^{(p)}$. Each $a_{i j}, i \neq j$, appears, possibly with the sign $-1,\left(\begin{array}{l}n-2 \\ p-1\end{array}\right)$ times as an off-diagonal element of $B^{(p)}$. In each row and each column of $B^{(p)} p(n-p)$ off-diagonal elements are of the form $\pm a_{i j}(i \neq j)$ and, for $2 \leqq p \leqq n-2$, the remaining off-diagonal elements are zeros. (2.1) implies also the following symmetry of the dependence of $B^{(p)}$ on $A$ : if, for $\alpha \neq \beta, b_{\alpha \beta}=a_{i j}, b_{\alpha \beta}=-a_{i j}$ or $b_{\alpha \beta}=0$ then $b_{\beta \alpha}=a_{j i}, b_{\beta \alpha}=-a_{j i}$ or $b_{\beta \alpha}=0$ respectively.

For $p=n,(2.1)$ gives $B^{(n)}(t)=b(1, \cdots, n \mid 1, \cdots, n)=\sum_{i=1}^{n} a_{i i}(t)$, and the differential system of the $n$th compound $\Delta(t)=C_{n}(Y(t))$ is Liouville's equation

$$
\Delta(t)^{\prime}=\left(\sum_{\imath=1}^{n} a_{i i}(t)\right) \Delta(t)
$$

We now consider the case $p=n-1$. Let $Y(t)$ be a fundamental solution of (1.2), then

$$
\begin{aligned}
\Delta(t)\left(Y(t)^{-1}\right)^{T} & =\left((-1)^{i+j} Y\left(\begin{array}{l}
1 \cdots i-1, i+1 \cdots n \\
1 \cdots j-1, j+1 \cdots n
\end{array}\right)\right)_{1}^{n} \\
& =\widetilde{C}_{n-1}(Y(t)) .
\end{aligned}
$$

Here the superscript $T$ denotes the transposition operation, and if $M=\left(m_{i j}\right)_{1}^{n}$ we define $\tilde{M}=\left(\widetilde{m}_{i j}\right)_{1}^{n}$ by

$$
\tilde{m}_{i j}=(-1)^{i+j} m_{n+1-i, n+1-j}, \quad i, j=1, \cdots, n \text {. }
$$

With this notation $\left[C_{n-1}(Y(t))\right]^{\prime}=B^{(n-1)}(t) C_{n-1}(Y(t))$ gives

$$
\left[\widetilde{C}_{n-1}(Y(t))\right]^{\prime}=\widetilde{B}^{(n-1)}(t) \widetilde{C}_{n-1}(Y(t)) \text {. }
$$

$\left(Y(t)^{-1}\right)^{T}$ is a solution of the system adjoint to (1.2):

$$
\left(Y(t)^{-1}\right)^{T \prime}=-A(t)^{T}\left(Y(t)^{-1}\right)^{T} .
$$

Differentiating (2.4) and using also (1.4), (2.5) and (2.6) we obtain

$$
-A(t)^{T}=\widetilde{B}^{(n-1)}(t)-\left(\sum_{i=1}^{n} a_{i i}(t)\right) I .
$$

(2.7) gives the connection between the adjoint equation and the equation for the $(n-1)$ st compound.

In the next section we use the following consequence of Theorem 1.

CoRollaRY 1. Let $A(t)=\left(a_{i j}(t)\right)_{1}^{n}$ and $B^{(p)}(t)=\left(b_{\alpha \beta}(t)\right)_{1}^{N}$ be the coefficient matrices of the system (1.2) and its compound systems, $1 \leqq p \leqq n$. Then,

(i) None of the matrices $B^{(p)}(t)$ contains elements of the form 
$-a_{i, i+1}(t),-a_{i+1, i}(t), i=1, \cdots, n-1$.

(ii) For each pair $(i, j),|i-j| \geqq 2, i, j=1, \cdots, n,-a_{i j}(t) \quad$ is an off-diagonal element of $B^{(2)}(t)$.

Proof. (i ) Formula (2.1) implies that $\pm a_{k, k+1}(t)$ and $\pm a_{k+1, k}(t)$, $k=1, \cdots, n-1$, can appear as elements of $B^{(p)}(t)$ only if they are an element $b\left(i_{1}, \cdots, i_{p} \mid j_{1}, \cdots, j_{p}\right)$, where $p-1$ of the indices of the two $p$-tuples $\alpha=\left(i_{1}, \cdots, i_{p}\right)$ and $\beta=\left(j_{1}, \cdots, j_{p}\right)$ coincide, but $i_{\ell} \neq j_{m}$, and where the set $\left\{i_{\ell}, j_{m}\right\}$ is the set $\{k, k+1\}$. If a given $(p-1)$ tuple of increasing indices, which contains neither $k$ nor $k+1$, is completed to a $p$-tuple of increasing indices by inserting $k$ or $k+1$, then it is necessary to insert either one of them at the same place, i.e., between the same two elements of the $(p-1)$-tuple. Hence $\ell=m$. and (2.1) implies (i).

(ii) If $1 \leqq i<i+2 \leqq j \leqq n$ then (2.1) gives $b(i, i+1 \mid i+1, j)=$ $-a_{i j}$; and if $1 \leqq j<j+2 \leqq i \leqq n$ then $b(j+1, i \mid j, j+1)=-a_{i j}$.

3. Positive, strictly positive, totally positive and strictly totally positive systems. Totally positive (TP) and strictly totally positive (STP) systems were defined in the introduction. To define positive and strictly positive systems we agree to call a real $n \times n$ matrix positive if all its elements are nonnegative; and the matrix is strictly positive if all its elements are positive. The differential system

$$
Y^{\prime}(t)=A(t) Y(t),
$$

is called positive in $(a, b)$, if for each pair $(r, t), a<r \leqq t<b, Y(t, r)$ is positive. (Here $Y(t)=Y(t, r)$ is the fundamental solution of (1.2) satisfying (1.5).) (1.2) is strictly positive in $(a, b)$ if for each pair $(r, t), a<r<t<b, Y(t, r)$ is strictly positive. We start with a criterion for the positivity of the system.

LemmA 1. Let the $n^{2}$ real functions $a_{i j}(t), i, j=1, \cdots, n$, be continuous in $(a, b),-\infty \leqq a<b \leqq \infty$, and set $A(t)=\left(a_{i j}(t)\right)_{1}{ }^{n}$. The differential system (1.2) is positive in $(a, b)$ if and only if all offdiagonal elements $a_{i j}(t), i \neq j, i, j=1, \cdots, n$, are nonnegative in $(a, b)$.

This lemma is known [1, p. 173, exercise 2]. For completeness, and also in view of the proof of the next lemma, we prove Lemma 1.

Proof. To show the necessity of the condition, suppose to the contrary that there exist indices $i^{*}$ and $j^{*}, i^{*} \neq j^{*}$, and a point $r$ in $(a, b)$ such that $a_{i^{*} j^{*}}(r)<0$. Let $Y(t, r)=\left(y_{i j}(t)\right)_{1}^{n}$ be the solution of (1.2) satisfying (1.5). Then $y_{i^{*} j^{*}}^{\prime}(r)=0$ and $y_{i^{*} j^{*}}(r)=a_{i^{*} j^{*}}(r)<0$. Hence, 
$y_{i^{*} j^{*}}(t)<0$ for all $t$ in some interval $(r, r+\varepsilon), \varepsilon>0$, and the system (1.2) is not positive.

We first prove sufficiency in the special case where all diagonal elements $a_{i i}(t)$ of $A(t)$ vanish identically in $(a, b)$. Each element of $A(t)$ is thus nonnegative, and the Peano-Baker expansion

$$
Y(t, r)=I+\int_{r}^{t} A(\tau) d \tau+\int_{r}^{t} A(\tau) \int_{r}^{\tau} A\left(\tau_{1}\right) d \tau_{1} d \tau+\cdots,
$$

shows that the same holds for each element of $Y(t, r), a<r \leqq t<b$.

To prove sufficiency in the general case (of arbitrary diagonal elements $a_{i i}(t)$ of $\left.A(t)\right)$ we choose a point $r, r \in(a, b)$, and define

$$
p_{i}(t, r)=\exp \int_{r}^{t} a_{i i}(\tau) d \tau, a<t<b, i=1, \cdots, n .
$$

Using these $n$ positive functions we now build the diagonal matrix

$$
P_{r}(t)=\operatorname{diag}\left(p_{1}(t, r), \cdots, p_{n}(t, r)\right), \quad a<t<b .
$$

If $Y(t)$ is an arbitrary solution of (1.2) we define $\widetilde{Y}_{r}(t)$ by

$$
Y(t)=P_{r}(t) \widetilde{Y}_{r}(t), \quad a<t<b .
$$

(1.2) and (3.2) to (3.4) imply that each $\widetilde{Y}_{r}(t)$ satisfies the equation

$$
\widetilde{Y}_{r}^{\prime}(t)=\widetilde{A}_{r}(t) \tilde{Y}_{r}(t), \quad a<t<b,
$$

where $\widetilde{A}_{r}(t)=\left(\widetilde{a}_{i j}(t, r)\right)_{1}^{n}$ is defined by

$$
\widetilde{a}_{i j}(t, r)=a_{i j}(t) \frac{p_{j}(t, r)}{p_{i}(t, r)}, i \neq j, i, j=1, \cdots, n, \quad a<t<b,
$$

and

$$
\widetilde{a}_{i i}(t, r)=0, i=1, \cdots, n, \quad a<t<b .
$$

The matrix $\widetilde{A}_{r}(t)$ has thus, together with the given matrix $A(t)$, nonnegative off-diagonal elements but its diagonal elements vanish identically. By the special case considered above, it follows that the system (3.5) is positive in $(a, b)$. Let now $\widetilde{Y}_{r}(t, r)$ be the fundamental solution $\widetilde{Y}_{r}(t)$ of $(3.5)$ which satisfies $\widetilde{Y}_{r}(r)=I$. Then $\widetilde{Y}_{r}(t, r)$ is positive for all $t$ in $[r, b)$. As $P_{r}=I$, it follows from (3.4) that

$$
Y(t, r)=P_{r}(t) \tilde{Y}_{r}(t, r),
$$

where $Y(t, r)$ is the solution of (1.2) satisfying (1.5). (3.8) implies that this matrix $Y(t, r)$ is positive for all $t$ in $[r, b)$. Since $r$ was arbitrary in $(a, b)$, this completes the proof of Lemma 1 .

For the next lemma it is convenient to use the following terminology. We denote the set of the $n^{2}$ elements $a_{i j}(t)$ of $A(t)$ by $S$. 
With each subset $F$ of $S$ we associate a matrix $C=\left(c_{i j}\right)_{1}^{n}$ in the following way: $c_{i j}=1$ if $a_{i j}(t) \in F, c_{i j}=0$ if $a_{i j}(t) \notin F$. Then we call $F$ irreducible or reducible if the associated matrix $C$ is, respectively, irreducible or reducible. If we associate with $F$ a directed graph $\Gamma$ of $n$ vertices $P_{1}, \cdots, P_{n}$, having a (directed) arc from $P_{i}$ to $P_{j}$ if and only if $a_{i j} \in F$, then $F$ is irreducible if and only if $\Gamma$ is strongly connected. (A matrix $C=\left(c_{i j}\right)_{1}^{n}$ is reducible if the index set $\{1, \cdots, n\}$ can be split into two nonvoid sets $\left\{i_{1}, \cdots, i_{\ell}\right\}$ and $\left\{j_{1}, \cdots, j_{m}\right\}, \iota+m=n$ such that $c_{i^{\lambda_{\mu}}}=0$ for $\lambda=1, \cdots, \iota, \mu=1, \cdots, m$. If no such partition of the index set exists, then $C$ is irreducible. A directed graph $\Gamma$ is strongly connected if and only if for every ordered pair $\left(P_{i}, P_{j}\right)$ of its vertices there exists a (directed) path leading from $P_{i}$ to $P_{j}$. The matrix $C$ is irreducible if and only if the corresponding graph $\Gamma$ is strongly connected. [9, pp. 18-20].)

Lemma 2. Let the $n^{2}$ real functions $a_{i j}(t), i, j=1, \cdots, n$, be continuous in $(a, b),-\infty \leqq a<b \leqq \infty$, and set $A(t)=\left(a_{i j}(t)\right)_{1}^{n}$. Let $S$ be the set of the $n^{2}$ functions $a_{i j}(t)$. For each $r, r \in(a, b)$, the subset $F(r)$ of $S$ is defined in the following way: $a_{i j}(t) \in F(r)$ if and only if $a_{i j}(t)$ does not vanish identically in any interval $[r, r+\varepsilon]$, $0<\varepsilon<b-r$. The differential system (1.2) is strictly positive in $(a, b)$ if and only if the following two conditions hold:

(a) Each off-diagonal element $a_{i j}(t), i \neq j, i, j=1, \cdots, n$, is nonnegative in $(a, b)$.

(b) For each $r, a<r<b$, the set $F(r)$ is irreducible.

Proof. The necessity of condition (a) follows from Lemma 1. We prove the necessity of (b) by negation and thus assume that there exists $r, r \in(a, b)$, such that $F(r)$ is reducible. As the graph $\Gamma(r)$ is thus not strongly connected it follows that there exists $\varepsilon, 0<\varepsilon<b-r$ and two indices $i^{*}, j^{*}, i^{*} \neq j^{*}$, such that for every given ordered set $\left(i_{0}, i_{1}, \cdots, i_{\ell}\right)$ of indices (with repetition), for which $i_{0}=i^{*}, i_{\ell}=j^{*}$, at least one function $a_{i_{\nu} i_{\nu}+1}(t), \nu=0, \cdots, \iota-1$, vanishes identically in $[r, r+\varepsilon]$. For $\iota=1$ this implies

$$
\int_{r}^{r+\varepsilon} a_{i^{*} * *}(\tau) d \tau=0
$$

For $\ell=2$ we obtain

$$
\int_{r}^{r+\varepsilon} \sum_{\nu=1}^{n} a_{i^{*} \nu}(\tau) \int_{r}^{\tau} a_{\nu j^{*}}\left(\tau_{1}\right) d \tau_{1} d \tau=0
$$

and similar equalities hold for $\iota \geqq 3$. Using these equalities it follows from (3.1) that the off-diagonal element $y_{i^{*} j^{*}}(r+\varepsilon, r)$ of the matrix $Y(r+\varepsilon, r)$ vanishes and $Y(r+\varepsilon, r)$ is thus not strictly positive. 
We prove sufficiency of conditions (a) and (b) again first in the special case where all diagonal elements $a_{i i}(t)$ of $A(t)$ vanish identically in $(a, b)$. By $(b)$, the set $F(r)$ is, for each $r \in(a, b)$, irreducible and in this special case $F(r)$ does not contain diagonal elements $a_{i i}(t)$. This and (a) imply that for any given $r, r \in(a, b)$, and any ordered pair $\left(i^{*}, j^{*}\right)$ of (not necessarily distinct) indices there exists an ordered set $\left(i_{0}, i_{1}, \cdots, i_{\ell}\right)$ of indices, $i_{0}=i^{*}, i_{\ell}=j^{*}$ and $i_{\nu} \neq i_{\nu+1}$ for $\nu=0, \cdots$, $\ell-1$, such that

$$
\int_{r}^{t} a_{i_{\nu} i_{\nu+1}}(\tau) d \tau>0
$$

for all $t$ in $(r, b)$ and all $\nu, \nu=0, \cdots, \iota-1$. But this implies that for all such $t$

$$
\int_{r}^{t} a_{i^{*} i_{1}}(\tau) \int_{r}^{\tau} a_{i_{1} i_{2}}\left(\tau_{1}\right) \cdots \int_{r}^{\tau \ell-2} a_{i_{\ell-1} j^{*}}\left(\tau_{\ell-1}\right) d \tau_{\ell-1} \cdots d \tau_{1} d \tau>0,
$$

and it follows that the element in the place $\left(i^{*}, j^{*}\right)$ of the $(\ell+1)$ th summand of the r.h.s. of (3.1) is, for $t \in(r, b)$, positive. As $r$ and the pair of indices were arbitrary it follows that the system (1.2) is, in this special case, strictly positive in $(a, b)$.

The sufficiency of conditions (a) and (b) in the general case (of arbitrary diagonal elements $a_{i i}(t)$ of $A(t)$ ) follows again by reduction to the special case (formulas (3.2) to (3.8)). We now use also the fact that if the set $F(r)$ is irreducible, so is the set $\widetilde{F}(r)$ which is obtained from $F(r)$ by deletion of its diagonal elements and by multiplication of its off-diagonal elements with positive functions. This completes the proof of Lemma 2.

These criteria for positivity and strict positivity and the corollary of $\S 2$ lead to the main results of this section.

TheOREm 2. Let the $n^{2}$ real functions $a_{i j}(t), i, j=1, \cdots, n$, be continuous in $(a, b),-\infty \leqq a<b \leqq \infty$, and set $A(t)=\left(a_{i j}(t)\right)_{1}^{n}$. The differential system

$$
Y^{\prime}(t)=A(t) Y(t),
$$

is $\mathrm{TP}$ in $(a, b)$ if and only if the following two conditions hold:

(a) $a_{i j}(t)=0,|i-j| \geqq 2, i, j=1, \cdots, n, a<t<b$.

(b) $\quad a_{i, i+1}(t) \geqq 0, a_{i+1, i}(t) \geqq 0, i=1, \cdots, n-1, a<t<b$.

Proof. As total positivity of the system (1.2) implies its positivity, it follows from Lemma 1 that all off-diagonal elements $a_{i j}(t), i \neq j$, of $A(t)$ have to be nonnegative in $(a, b)$. If an element $a_{i j}(t),|i-j| \geqq 2$, 
were to be positive for some $t$ part (ii) of Corollary 1 would imply that the matrix $B^{(2)}(t)$ of the second compound system has an off-diagonal element which is somewhere negative, and Lemma 1, applied to this second compound system, then shows that (1.2) is not TP. Conditions (a) and (b) are thus necessary. Their sufficiency follows from part (i) of Corollary 1 and the sufficiency part of Lemma 1, applied to all compound systems (1.3). (We remark that we also use that the $p$ th compound of the unit matrix $I=\left(\delta_{i j}\right)_{1}^{n}$ is again $I=\left(\delta_{\alpha \beta}\right)_{1}^{N}$. Hence if $Y(t)=Y(t, r)$ is the solution of (1.2) which satisfies (1.5), then its compound also satisfies $C_{p}(Y(r))=I$.)

Theorem 3. Let the $n^{2}$ real functions $a_{i j}(t), i, j=1, \cdots, n$, be continuous in $(a, b),-\infty \leqq a<b \leqq \infty$, and set $A(t)=\left(a_{i j}(t)\right)_{1}^{n}$. The differential system

$$
Y^{\prime}(t)=A(t) Y(t),
$$

is STP in $(a, b)$ if and only if the following three conditions hold:

(a) $a_{i j}(t)=0,|i-j| \geqq 2, i, j=1, \cdots, n, \quad a<t<b$.

(b) $\quad a_{i, i+1}(t) \geqq 0, a_{i+1, i}(t) \geqq 0, i=1, \cdots, n-1, a<t<b$.

(c) None of the $2 n-2$ functions mentioned in (b) vanishes identically in any interval $[r, s], a<r<s<b$.

Proof. The necessity of conditions (a) and (b) follows from Theorem 2. To prove that condition (c) is necessary, we consider the $(0,1)$ matrix $C^{*}=\left(c_{i j}^{*}\right)_{1}^{n}$ where $c_{i j}^{*}=0$ if $|i-j| \neq 1$, and $c_{i j}^{*}=1$ if $|i-j|=1$. Then the following statement holds. (i) $C^{*}$ is irreducible, and (ii) if any element equal to 1 of $C^{*}$ is replaced by 0 then the new matrix is reducible. This is easily seen by considering the corresponding directed graph $\Gamma^{*}$. Assume now that condition (c) is not satisfied and that one of the $2 n-2$ functions $a_{i, i+1}(t)$ and $a_{i+1, i}(t)$ vanishes identically in a certain interval $[r, s]$. Part (ii) of the italicized statement implies that the set $F(r)$, defined in Lemma 2, is reducible and Lemma 2 implies that the system (1.2) is not strictly positive in $(a, b)$. This contradicts the assumption of the present theorem and condition (c) is thus necessary.

To prove the sufficiency of conditions (a) to (c), we consider also the $(0,1)$ matrices $C^{*(p)}, 1 \leqq p \leqq n$, which are built from the elements $c_{i j}^{*}$ of $C^{*}=C^{*(1)}$ by the rule (2.1). Namely,

$$
C^{*(p)}=\left(c_{\alpha \beta}^{*}\right)_{1}^{N}, N=\left(\begin{array}{c}
n \\
p
\end{array}\right) \quad \text { and } \quad c_{\alpha \beta}^{*}=c^{*}\left(i_{1}, \cdots, i_{p} \mid j_{1}, \cdots, j_{p}\right)=0
$$

except if exactly $p-1$ of the indices of $\alpha$ coincide with $p-1$ indices of $\beta$ and the two remaining indices satisfy $\left|i_{\ell}-j_{\ell}\right|=1$; in this 
case $c_{\alpha \beta}^{*}=1$. For each $p, 1 \leqq p \leqq n, C^{*(p)}$ is irreducible. (For $p=1$ this is part (i) of the former italicized statement.) This is again easily seen by considering the corresponding graph $\Gamma^{*(p)} \cdot\left(\Gamma^{*(p)}\right.$ has $N$ vertices $P_{\alpha}=P\left(i_{1}, \cdots, i_{p}\right), P_{\beta}=P\left(j_{1}, \cdots, j_{p}\right)$, etc. There are arcs (in both directions) between $P_{\alpha}$ and $P_{\beta}$ if $p-1$ of the indices of $\alpha$ and $\beta$ coincide and $\left|i_{\ell}-j_{\ell}\right|=1$. Clearly there exists a path of length $\sum_{y=1}^{p}\left(i_{\nu}-\nu\right)$ leading from $P_{\alpha}$ to the first vertex $P_{\alpha^{*}}\left(\alpha^{*}=1=\right.$ $(1, \cdots, p)$ ) and similarly there exists a path leading from $P_{\alpha^{*}}$ to $P_{\beta}$. $\Gamma^{*(p)}$ is thus strongly connected). Using part (i) of Corollary 1 and the irreducibility of $C^{*(p)}, 1 \leqq p \leqq n$, it follows that the present conditions (a) to (c) imply the validity of conditions (a) and (b) of Lemma 2 for each compound system (1.3). Each of these systems is therefore strictly positive in $(a, b)$ and (1.2) is thus STP. This completes the proof of Theorem 3 .

4. Vector solutions of strictly totally positive systems. Our next result refers to the number of sign changes of a given nontrivial vector solution $y(t)$ of a STP system (1.1). We use the standard notation [2,4]. If $x=\left(x_{1}, \cdots, x_{n}\right)$ is a real vector, $x \neq 0$, then $S^{-}(x)$ denotes the number of sign changes in the sequence obtained from $x_{1}, x_{2}, \cdots, x_{n}$ by deleting all zero terms; $S^{+}(x)$ denotes the maximum number of sign changes possible by allowing each zero to be replaced \pm 1 (or equivalently, $S^{+}(x)=\varlimsup_{y \rightarrow x} S^{-}(y)$ ).

\section{THEOREM 4. (i) Let the differential system}

$$
y^{\prime}(t)=A(t) y(t),
$$

be $\operatorname{STP}$ in $(a, b),-\infty \leqq a<b \leqq \infty$ and let $y(t)$ be a nontrivial solution. Then

$$
S^{\dagger}(y(s)) \leqq S^{-}(y(r)) \quad \text { for all }(r, s) \text { satisfying } a<r<s<b .
$$

(ii) Conversely, if (4.1) is valid for every nontrivial solution $y(t)$ of the system (1.1), then this system is $\mathrm{STP}$ in $(a, b)$.

Proof. (i) Let $Y(t)=Y(t, r)$ be the fundamental solution of

$$
Y^{\prime}(t)=A(t) Y(t),
$$

satisfying

$$
Y(r)=I
$$

For all $s$ and $r$ in $(a, b)$

$$
y(s)=Y(s, r) y(r) .
$$


By assumption the matrix $Y(s, r)$ is, for $r<s$, STP. (4.1) follows from the variation-diminishing property of such matrices [4, p. 219, Th. 1.2 , (a)].

(ii) Let the index $k, 1 \leqq k \leqq n$, and the point $r, r \in(a, b)$, be given and consider nontrivial solutions $y(t)$ of (1.1) which satisfy

$$
y_{k}(r)=0 \text {. }
$$

(4.2) and (4.3) give

$$
y(s)=Y_{k}(s, r) c .
$$

Here $c$ is the $(n-1)$ vector $\left(y_{1}(r), \cdots, y_{k-1}(r), y_{k+1}(r), \cdots, y_{n}(r)\right)$ and $Y_{k}(s, r)$ is the $n \times(n-1)$ matrix obtained from $Y(s, r)$ by deletion of the $k$ th column. By assumption (4.1), we have for $r<s$,

$$
S^{\leftarrow}(y(s)) \leqq S^{-}(y(r))=S^{-}(c) \text {. }
$$

As this holds for every nonnull vector $c$, it follows that $Y_{k}(s, r)$ is, for $r<s$, strictly sign-regular of order $n-1$ [4, p. 219, Th. 1.2, (b)]; i.e., all minors of $Y_{k}(s, r)$ are nonzero and, for each $p, 1 \leqq p \leqq n-1$, all minors of order $p$ have the same sign, possibly dependent on $p$. But as $Y(r, r)=I$, it follows that $Y_{k}(r, r)$ has for each $p, 1 \leqq p \leqq$ $n-1$, a minor equal to 1 . It follows, by continuity, that all minors of $Y_{k}(s, r), r<s$, are positive. As $k$ was an arbitrary index, this implies that all minors, up to the order $n-1$, of $Y(s, r)$ are positive for $r<s$. But the determinant of $Y(s, r)$ is always positive and we have thus proved that the system (1.1) is STP.

We remark that by the last two theorems property (4.1), for all nontrivial solutions $y(t)$, is equivalent to the properties (a) to (c) of $A(t)$ stated in Theorem 3. A direct proof of this equivalence, without use of the variation-diminishing properties of the STP matrix $Y(s, r)$, seems to be rather tedious.

The next theorem, and the examples in the final section, will give some information about the number of points at which each component of a fixed solution of an STP system (1.1) may vanish. It might be of interest to consider here briefly the case of such systems with constant coefficients $A(t)=A$. $A$ is thus a Jacobi matrix with positive off-diagonal elements. But the class of Jacobi matrices $B$ with negative off-diagonal elements was studied in detail by Gantmacher and Krein [2, Ch. $2, \S 1$.$] . For A(=-B)$ it follows that $A$ has $n$ distinct real characteristic values $\lambda_{j}, \lambda_{1}<\lambda_{2}<\cdots<\lambda_{n}$, (and that for the characteristic vector $u^{(j)}=\left(u_{i j}, \cdots, u_{n j}\right)$, corresponding to $\lambda_{j}, S^{+}\left(u^{(j)}\right)=$ $\left.S^{-}\left(u^{(j)}\right)=n-j, j=1, \cdots, n\right)$. Every solution $y(t)$ of the corresponding system (1.1) is therefore of the form 


$$
y_{i}(t)=\sum_{j=1}^{n} c_{j} u_{i j} e^{\lambda_{j} t}, \quad i=1, \cdots, n,
$$

and it follows that in this case each component $y_{i}(t)$ of a nontrivial solution $y(t)$ vanishes at most $n-1$ times. (Note that for any system (1.1) there always exist nontrivial solutions $y(t)$ satisfying $(n-1)$ homogeneous conditions.) As already mentioned in the introduction a more precise statement holds for the total number of zeros of $y_{1}(t)$ and $y_{n}(t)$ for any STP system (Theorem 5, (ii)); and the examples will show that, for any $n, n \geqq 3$, there exist STP systems with variable $A(t)$ having a solution $y(t)$ for which each interior component $y_{i}(t)$, $i=2, \cdots, n-1$, vanishes infinitely many times in $(-\infty, \infty)$.

To facilitate the proof of Theorem 5 we now state some evident properties of the functions $S^{+}$and $S^{-}$as a lemma.

Lemma 3. Let $x=\left(x_{1}, \cdots, x_{n}\right)$, be a real nonnull vector. Then

$$
0 \leqq S^{-}(x) \leqq S^{+}(x) \leqq n-1 .
$$

If $m$ components of $x$ vanish, $1 \leqq m \leqq n-1$, then

$$
S^{+}(x) \geqq m, S^{-}(x) \leqq n-m-1 \text {. }
$$

If $x_{1}=0$, or if $x_{n}=0$, then

$$
S^{+}(x)-S^{-}(x) \geqq 1 .
$$

If $x_{1}=0$ and $x_{n}=0$, then

$$
S^{+}(x)-S^{-}(x) \geqq 2 \text {. }
$$

Part (i) of Theorem 4, and Lemma 3, now imply the following theorem.

THEOREM 5. Let the differential system

$$
y^{\prime}(t)=A(t) y(t)
$$

be $\operatorname{STP}$ in $(a, b),-\infty \leqq a<b \leqq \infty$, and let $y(t)=\left(y_{1}(t), \cdots, y_{n}(t)\right)$ be a nontrivial solution.

(i ) If $S^{-}(y(r))=0, r \in(a, b)$, then no component of $y(t)$ vanishes in $(r, b)$. If $S^{+}(y(s))=n-1, s \in(a, b)$, then no component of $y(t)$ vanishes in $(a, s)$.

(ii) Let $k$ and $<$ be nonnegative integers and assume that

$$
y_{1}\left(\alpha_{i}\right)=0, i=1, \cdots, k, a<\alpha_{1}<\cdots<\alpha_{k}<b,
$$

and that

$$
y_{n}\left(\beta_{j}\right)=0, j=1, \cdots, \iota, a<\beta_{1}<\cdots<\beta_{\ell}<b .
$$


Then $k+\ell \leqq n-1$. Moreover, if $k+\ell=n-1$, then no component of $y(t)$ vanishes in $\left(a, \min \left(\alpha_{1}, \beta_{1}\right)\right) \cup\left(\max \left(\alpha_{k}, \beta_{\ell}\right), b\right)$.

(iii) Assume that $m$ components of $y(r), r \in(a, b)$, vanish, and that

$$
y_{1}\left(\alpha_{i}\right)=0, i=1, \cdots, k, r<\alpha_{1}<\cdots<\alpha_{k}<b,
$$

and that

$$
y_{n}\left(\beta_{j}\right)=0, j=1, \cdots, \iota, r<\beta_{1}<\cdots<\beta_{\ell}<b .
$$

Then $k+\iota \leqq n-m-1$. Moreover, if $k+\iota=n-m-1$, then no component of $y(t)$ vanishes in $\left(\max \left(\alpha_{k}, \beta \ell\right), b\right) . \quad A$ similar statement holds for the number of zeros of $y_{1}(t)$ and $y_{n}(t)$ in $(a, r)$.

Proof. (i ) $S^{-}(y(r))=0$ and (4.1) imply $S^{+}(y(t))=0, r<t<b$, and the first inequality of (4.6) implies that no component of $y(t)$ vanishes. $S^{+}(y(s))=n-1$ and (4.1) imply $S^{-}(y(t))=n-1, a<t<s$, and the other inequality of (4.6) gives the desired conclusion.

(ii) Denote the union of the sets $\left\{\alpha_{i}\right\}_{1}^{k}$ and $\left\{\beta_{j}\right\}_{1}^{<}$by

$$
\left\{t_{\nu}\right\}_{1}^{p}, t_{1}<\cdots<t_{p},(\max (k, \iota) \leqq p \leqq k+\ell) .
$$

Then

$$
\begin{aligned}
k+\ell & \leqq \sum_{\nu=1}^{p}\left[S^{+}\left(y\left(t_{\nu}\right)\right)-S^{-}\left(y\left(t_{\nu}\right)\right)\right] \\
& =\sum_{\nu=2}^{p}\left[S^{+}\left(y\left(t_{\nu}\right)\right)-S^{-}\left(y\left(t_{\nu-1}\right)\right)\right]+S^{+}\left(y\left(t_{1}\right)\right)-S^{-}\left(y\left(t_{p}\right)\right) \\
& \leqq S^{+}\left(y\left(t_{1}\right)\right)-S^{-}\left(y\left(t_{p}\right)\right) \leqq n-1 .
\end{aligned}
$$

Here the first inequality sign follows from (4.7) and (4.8), the second inequality sign follows from (4.1) and the last one from (4.5). This proves the main assertion of (ii). If $k+\ell=n-1$, then (4.9) implies $S^{-}\left(y\left(t_{p}\right)=0\right.$ and $S^{+}\left(y\left(t_{1}\right)\right)=n-1$ and the remaining assertion of (ii) now follows from (i).

(iii) Let $t_{1}, \cdots, t_{p}$ have the same meaning as above. (4.9), the assumption $r<t_{1}$ and (4.1), and (4.6) give

$$
\begin{aligned}
k+\ell & \leqq S^{+}\left(y\left(t_{1}\right)\right)-S^{-}\left(y\left(t_{p}\right)\right) \\
& \leqq S^{-}(y(r))-S^{-}\left(y\left(t_{p}\right)\right) \leqq n-m-1 .
\end{aligned}
$$

If $k+\ell=n-m-1$, then (4.10) and $S^{-}(y(r) \leqq n-m-1$ imply $S^{-}\left(y\left(t_{p}\right)\right)=0$ and no component of $y(t)$ vanishes in $\left(t_{p}, b\right)$. For zeros to the left of $r, a<t_{1}<\cdots<t_{p}<r$, we obtain

$$
\begin{aligned}
k+\ell & \leqq S^{+}\left(y\left(t_{1}\right)\right)-S^{-}\left(y\left(t_{p}\right)\right) \\
& \leqq S^{+}\left(y\left(t_{1}\right)\right)-S^{+}(y(r)) \leqq n-m-1 .
\end{aligned}
$$

If $k+\ell=n-m-1$ this gives $S^{+}\left(y\left(t_{1}\right)\right)=n-1$ and no component vanishes in $\left(a, t_{1}\right)$. This completes the proof of Theorem 5 . 
We remark that the constants $n-1$ of part (ii) and $n-m-1$ of part (iii) of this theorem, are the best possible as there always exist nontrivial solutions of (1.1) satisfying $n-1$ conditions $y_{i_{\nu}}\left(t_{\nu}\right)=$ $0,1 \leqq i_{\nu} \leqq n, a<t_{\nu}<b, \nu=1, \cdots, n-1$. We conclude this section with another direct consequence of (4.1). Let $r$ and $s$ be given points, $a<r<s<b$, and assume that $y(t)$ is a nontrivial solution of (1.1) such that $k$ components of $y(r)$ and $\angle$ components of $y(s)$ vanish. Then $k+\ell \leqq n-1$. Moreover, if $k+\ell=n-1$, then there exists-except for a multiplicative constant-precisely one nontrivial solution $y(t)$ of (1.1) satisfying the given set of conditions $y_{i_{\nu}}(r)=0, y_{j_{\mu}}(s)=0, \nu=$ $1, \cdots, k, \mu=1, \cdots, \iota$. To prove the first part, we remark that, by (4.6), $S-(y(r)) \leqq n-k-1$ and $S^{+}(y(s)) \geqq \ell$. (4.1) gives therefore $k+\ell \leqq$ $n-1$. Assume now $k+\ell=n-1$ and let $y(t)$ and $u(t)$ be two solutions satisfying the given set of $(n-1)$ conditions. We can then form a linear combination $v(t)=c_{1} y(t)+c_{2} u(t)$ such that $k+1$ components of $v(r)$ and the former $l=n-k-1$ components of $v(s)$ vanish. $v(t)$ violates the first part of the above statement unless it reduces to the trivial solution. Hence $u(t)=c y(t)$ (cf. [7, p. 507]). This statement can also be obtained directly from the strict total positivity of the matrix $Y(s, r)$.

5. Vector solutions of totally positive systems. According to Theorem 4, the inequality (4.1) is characteristic for STP systems. It follows from (4.1) that $S^{-}(y(t))$ and $S^{+}(y(t))$ are decreasing functions of $t$. These consequences of (4.1) characterize the larger class of TP systems.

THeorem 6. (i) Let the differential system

$$
y^{\prime}(t)=A(t) y(t),
$$

be TP in $(a, b),-\infty \leqq a<b \leqq \infty$, and let $y(t)$ be a nontrivial solution. Then

$$
S^{-}(y(s)) \leqq S^{-}(y(r)) \quad \text { for all }(r, s) \text { satisfying } a<r \leqq s<b,
$$

and

(5.2) $\quad S^{+}(y(s)) \leqq S^{\top}(y(r))$ for all $(r, s)$ satisfying $a<r \leqq s<b$.

(ii) Conversely, if (5.1) is valid for every nontrivial solution. $y(t)$ of the system (1.1), or if (5.2) is valid for every $y(t)$, then the system (1.1) is TP in $(a, b)$.

Proof. ( i ) We obtain the necessity of (5.1) and (5.2) by an approximation procedure. Let the constant matrix $C^{*}=\left(c_{i j}^{*}\right)_{1}^{n}$ be defined as in the proof of Theorem $3\left(c_{i j}^{*}=1\right.$ if $|i-j|=1$, otherwise 
$\left.c_{i j}^{*}=0\right)$. If the system (1.1) is TP in $(a, b)$, then it follows from Theorems 2 and 3 that the system

$$
y_{s}^{\prime}(t)=A_{s}(t) y_{\varepsilon}(t), \quad A_{\varepsilon}(t)=A(t)+\varepsilon C^{*},
$$

is, for $\varepsilon>0$, STP in $(a, b)$. To prove (5.1) let the solution $y(t)$ of (1.1) and the point $r$ be given. For any $\varepsilon>0$, let $y_{\varepsilon}(t)$ be the solution of (5.3) satisfying

$$
y_{\varepsilon}(r)=y(r) \text {. }
$$

(4.1) and (5.4) imply that for any $\varepsilon>0$, and for any $s, s \in(r, b)$,

$$
S^{+}\left(y_{\varepsilon}(s)\right) \leqq S^{-}(y(r)) \text {. }
$$

By a standard theorem on differential equations (cf. [3, p. 55, Corollary 4.1])

$$
\lim _{\varepsilon \rightarrow 0} y_{\varepsilon}(s)=y(s)
$$

This and the relation

$$
S^{-}\left(\lim _{\varepsilon \rightarrow 0} y_{\varepsilon}(s)\right) \leqq \lim _{\varepsilon \rightarrow 0} S^{+}\left(y_{\varepsilon}(s)\right),
$$

[4, p. 217, Lemma 1.1] imply

$$
S^{-}(y(s)) \leqq \lim _{\varepsilon \rightarrow 0} S^{+}\left(y_{\varepsilon}(s)\right) .
$$

(5.5) and (5.6) imply (5.1).

To obtain (5.2) let the solution $y(t)$ of (1.1) and the point $s$ be given. For any $\varepsilon>0$, let $\widetilde{y}_{\varepsilon}(t)$ be the solution of (5.3) satisfying

$$
\widetilde{y}_{\mathrm{s}}(s)=y(s) \text {. }
$$

(4.1) and $\left(5.4^{\prime}\right)$ imply that for any $\varepsilon>0$, and for any $r, r \in(a, s)$

$$
S^{+}(y(s)) \leqq S^{-}\left(\widetilde{y}_{s}(r)\right) \text {. }
$$

For $\varepsilon \rightarrow 0$,

$$
\varlimsup_{\varepsilon \rightarrow 0} S^{-}\left(\widetilde{y}_{\varepsilon}(r)\right) \leqq S^{+}(y(r)) .
$$

(5.5') and (5.6') imply (5.2). This completes the proof of part (i). (We remark that (5.1) follows also directly from a theorem of Schoenberg [8, Satz 1] (cf. [2, p. 290] and [4, p. 21]) applied to the vector equation (4.2). Moreover (5.1) and (5.2) are equivalent as we shall show in Lemma 4.)

(ii) To prove the first half of this converse assertion, we assume the validity of (5.1) for all nontrivial solutions $y(t)$ of (1.1). We now 
proceed as in the proof of part (ii) of Theorem 4. The index $k$ and the point $r$ are fixed and we consider only nontrivial solutions of (1.1) which satisfy

$$
y_{k}(r)=0 \text {. }
$$

Defining $c$ as before and now using $S^{-}(y(s)) \leqq S^{-}(c), r<s$, we find that the $n \times(n-1)$ matrix $Y_{k}(s, r)$ (which is of rank $n-1$ ) is, for $r<s$, sign-regular of order $n-1$ [4, p. 222, Th. 1.4]; i.e., for each $p, 1 \leqq p \leqq n-1$, all nonvanishing minors of order $p$ of $Y_{k}(s, r)$ have the same sign. But, for each $p, Y_{k}(r, r)$ has a positive minor of this order and not all minors of order $p$ of $Y_{k}(s, r)$ can vanish. It follows, by continuity, that all minors of $Y_{k}(s, r)$ are nonnegative for $r<s$ and we thus proved the first half of (ii). (This follows again directly from the converse theorem of Schoenberg [8, Satz 2]). The second half of (ii) follows from the first half and the following lemma.

Lemma 4. Let the $n^{2}$ real functions $a_{i j}(t), i, j=1, \cdots, n$, be continuous in $(a, b),-\infty \leqq a<b \leqq \infty$ and set $A(t)=\left(a_{i j}(t)\right)_{1}^{n}$ and let (1.1) be the corresponding differential system. If, for each nontrivial solution $y(t), S^{-}(y(t))$ is a decreasing function of $t$ in $(a, b)$, then the same holds for $S^{+}(y(t))$. Conversely, if $S^{+}(y(t))$ is, for each nontrivial solution $y(t)$, a decreasing function of $t$, then the same holds for $S^{-}(y(t))$.

Proof. We shall use Theorem 2 and the (already proved) parts of Theorem 6 relating to (5.1), i.e., the first half of part (i) and the first half of part (ii). Let $y(t)$ be a nontrivial solution of (1.1) and define $y^{*}(t)=\left(y_{1}^{*}(t), \cdots, y_{n}^{*}(t)\right)$ by

$$
y_{i}^{*}(t)=(-1)^{i} y_{i}(t), i=1, \cdots, n, \quad a<t<b .
$$

This and (1.1) imply that

$$
\frac{d y^{*}}{d t}=B(t) y^{*}(t), \quad a<t<b,
$$

where $B(t)=\left(b_{i j}(t)\right)_{1}^{n}$ is given by

$$
b_{i j}(t)=(-1)^{i+j} a_{i j}(t), i, j=1, \cdots, n, \quad a<t<b .
$$

We now define

$$
u(\tau)=y^{*}(-\tau), \quad-b<\tau<-a,
$$

and

$$
C(\tau)=-B(-\tau), \quad-b<\tau<-a .
$$


(5.8), (5.10) and (5.11) give

$$
\frac{d u}{d \tau}=C(\tau) u(\tau), \quad-b<\tau<-a .
$$

(5.7) and (5.10) imply, that for each $t, a<t<b$,

$$
S^{+}(y(t))=(n-1)-S^{-}\left(y^{*}(t)\right)=(n-1)-S^{-}(u(-t)) .
$$

We now assume that $S-(y(t))$ is, for each $y(t)$, a decreasing function of $t$. By the first half of Theorem 6, (ii), and by Theorem 2, it follows that $A(t)$ is a Jacobi matrix with nonnegative off-diagonal elements. (5.9) and (5.11) show that the same holds for $C(\tau)$, hence using once more Theorem 2 and the first half of Theorem 6, (i), it follows that $S^{-}(u(\tau))$ is a decreasing function of $\tau ; S^{-}\left(y^{*}(t)\right)$ is thus an increasing function of $t$, and (5.13) implies that $S^{+}(y(t))$ is a decreasing function of $t$. Conversely, assume that $S^{+}(y(t))$ is, for each $y(t)$, a decreasing function of $t . \quad S^{-}(u(\tau))$ is then also a decreasing function of $\tau, C(\tau)$ is a Jacobi matrix with nonnegative off-diagonal elements, and the same holds for $A(t)$. $S^{-}(y(t))$ decreases therefore for each $y(t)$. This proves Lemma 4 and we have thus completed the proof of Theorem 6 .

(We shall use formulas (5.7) to (5.13) in the proof of the following lemma. We remark here that Lemma 4 is only a special case of the following statement: If the real $n \times n$ matrix $M$ is nonsingular, and if for every pair of nonnull vectors $(x, z), z=M x, S^{-}(z) \leqq S^{-}(x)$, then $S^{+}(z) \leqq S^{+}(x)$ holds also for all these pairs. This follows easily from the above mentioned theorems of Schoenberg, by obvious analogues. of (5.7) and (5.9) and a well-known formula for the minors of the inverse matrix $[4$, p. 5].)

For the proof of our final theorem we need the following lemma.

Lemma 5. Let the differential system

$$
y^{\prime}(t)=A(t) y(t)
$$

be TP in $(a, b),-\infty \leqq a<b \leqq \infty$, let $y(t)=\left(y_{1}(t), \cdots, y_{n}(t)\right)$ be $a$ nontrivial solution, and let the points $r$ and satisfy $a<r<s<b$.

( i) If

$$
y_{1}(r)=0, y_{1}(s) \neq 0
$$

or if

$$
y_{n}(r)=0, y_{n}(s) \neq 0,
$$




$$
S^{+}(y(r))-S^{+}(y(s)) \geqq 1 .
$$

Moreover, if both (5.14) and (5.15) hold, then

$$
S^{+}(y(r))-S^{+}(y(s)) \geqq 2 .
$$

(ii) If

$$
y_{1}(r) \neq 0, y_{1}(s)=0 \text {, }
$$

or if

$$
y_{n}(r) \neq 0, y_{n}(s)=0 \text {, }
$$

then

$$
S^{-}(y(r))-S^{-}(y(s)) \geqq 1 .
$$

Moreover, if both $\left(5.14^{\prime}\right)$ and $\left(5.15^{\prime}\right)$ hold, then

$$
S^{-}(y(r))-S^{-}(y(s)) \geqq 2 .
$$

Proof. ( i ) We assume that (5.14) holds for a given pair $(r, s)$, $a<r<s<b$. By the continuity of $y(t)$, and by considering, if necessary, $-y(t)$ instead of $y(t)$, it follows that there exist points $\left(r_{1}, s_{1}\right), r \leqq r_{1}<s_{1} \leqq s$, such that

$$
y\left(r_{1}\right)=0, \quad \text { and } y_{1}(t)>0 \text { for all } t \text { in }\left(r_{1}, s_{1}\right] \text {, }
$$

and such that no component $y_{i}(t)$ for which $y_{i}\left(r_{1}\right) \neq 0$ vanishes in $\left[r_{1}, s_{1}\right]$. We now consider the possible values of $y_{2}\left(r_{1}\right)$. (a) If $y_{2}\left(r_{1}\right)>0$, then our choice of $\left[r_{1}, s_{1}\right]$ implies that also $y_{2}\left(s_{1}\right)>0$. The pair $\left(y_{1}(t)\right.$, $\left.y_{2}(t)\right)$ contributes in this case to $S^{+}\left(y\left(r_{1}\right)\right)$ and gives no contribution to $S^{+}\left(y\left(s_{1}\right)\right)$, and the remaining pairs $\left(y_{i}(t), y_{i+1}(t)\right), i=2, \cdots, n-1$, cannot contribute more to $S^{+}\left(y\left(s_{1}\right)\right)$ than to $S^{+}\left(y\left(r_{1}\right)\right)$. Hence, in this case,

$$
S^{+}\left(y\left(r_{1}\right)\right)-S^{+}\left(y\left(s_{1}\right)\right) \geqq 1 .
$$

(b) The assumption $y_{2}\left(r_{1}\right)<0$ implies $y_{2}\left(s_{1}\right)<0$. These inequalities and (5.18), and once more, the fact that components which are $\neq 0$ at $r_{1}$ remain so in $\left[r_{1}, s_{1}\right]$, give $S^{-}\left(y_{1}\left(r_{1}\right)\right)<S^{-}\left(y_{1}\left(s_{1}\right)\right)$. This contradicts (5.1) and this case is thus excluded. There remains the case (c) $y_{2}\left(r_{1}\right)=0 . \quad y_{2}(t)$ cannot vanish identically in $\left[r_{1}, s_{1}\right]$ as then the first component of the equation (1.1), i.e., $y_{1}^{\prime}=a_{11} y_{1}+a_{12} y_{2}$ contradicts (5.18). Furthermore, $y_{2}(t)$ cannot become negative in $\left(r_{1}, s_{1}\right]$, as $y_{2}\left(r_{1}\right)=$ $0, y_{2}(t)<0$ and (5.18) would again give $S^{-}\left(y\left(r_{1}\right)\right)<S^{-}(y(t)), r<t$. Hence there exists a point $s_{2}, s_{2} \in\left(r_{1}, s_{1}\right]$ such that $y_{2}\left(s_{2}\right)>0$, and we obtain

$$
S^{+}\left(r_{1}\right)-S^{+}\left(s_{2}\right) \geqq 1 \text {. }
$$


As $r \leqq r_{1}<s_{2} \leqq s_{1} \leqq s, \quad(5.19),\left(5.19^{\prime}\right)$ and (5.2) imply (5.16). The assumption (5.15) gives the same conclution. As $t$ increases from $r$ to $s$, the decrease of $S^{+}(y(t))$ is, under the assumption (5.14) due to the pair $\left(y_{1}(t), y_{2}(t)\right)$. Under the assumption (5.15), it is due to the pair $\left(y_{n-1}(t), y_{n}(t)\right)$, and it therefore follows that the simultaneous validity of (5.14) and (5.15) implies (5.17).

(ii) This part now follows from part (i) by the previously used transformation (formulas (5.7) to (5.13)). Together with the system (1.1) also the system (5.12) is TP. $\left(5.14^{\prime}\right)$ becomes $u_{1}(-s)=0, u_{1}(-r) \neq 0$ and part (i) gives $S^{+}(u(-s))-S^{+}(u(-r)) \geqq 1$. This and

$$
S^{+}(u(-t))=S^{+}\left(y^{*}(t)\right)=n-1-S^{-}(y(t))
$$

gives $\left(5.16^{\prime}\right)$ and we have thus completed the proof of the lemma.

In Theorem 5 we obtained results on the behavior of solutions $y(t)$ of a STP system (1.1). If the system (1.1) is TP, but not STP, then none of the assertions of Theorem 5 remains valid. To show this, let $A(t)=\left(a_{i j}(t)\right)_{1}^{n}$ be a Jacobi matrix with nonnegative off-diagonal elements in $(a, b)$ and assume that for a given index $q, 1 \leqq q \leqq n-1$, and a given interval $(\alpha, \beta), \alpha \leqq \alpha<\beta \leqq b$, the element $a_{q+1, q}(t)$ vanishes identically in $(\alpha, \beta)$. We now consider (1.1) only in this subinterval $(\alpha, \beta)$. Here (1.1) may be satisfied by solution vectors $y(t)$ for which $y_{q+1}(t) \equiv \cdots \equiv y_{n}(t) \equiv 0$. If we consider only such solutions $y(t)$, then the vector consisting of their first $q$ components $\widetilde{y}(t)=\left(y_{1}(t), \cdots, y_{q}(t)\right)$ satisfies an equation of the form

$$
\widetilde{y}^{\prime}(t)=\widetilde{A}(t) \widetilde{y}(t), \quad \alpha<t<\beta,
$$

where

$$
\widetilde{A}(t)=\left(a_{i j}(t)\right)_{1}^{q}, \quad \alpha<t<\beta .
$$

This $q$ th order system (5.21) is again TP (possibly even STP) in $(\alpha, \beta)$, and we obtain a $q$-dimensional subspace of the solutions of (1.1) by adding the $n-q$ zero components $y_{q+1}(t) \equiv \cdots \equiv y_{n}(t) \equiv 0$ to an arbitrary solution of (5.20). These solutions of (1.1) do not satisfy the assertions of Theorem 5. Indeed, let $r \in(\alpha, \beta)$ and choose $y_{1}(r)=$ $\cdots=y_{q}(r)=1$. Then $S^{-}(y(r))=0$, but the $n-q$ last components of $y(t)$ vanish identically in $(r, \beta)$. If we choose $y_{i}(r)=(-1)^{i}$, $i=1, \cdots, q$, then $S^{+}(y(r))=n-1$, but the last components vanish identically $(\alpha, r)$. This shows that part (i) of Theorem 5 is not valid for the present system. Parts (ii) and (iii) are not valid as $y_{n}(t) \equiv 0$ in $(\alpha, \beta)$. If we assume that an element of the first superdiagonal $a_{q, q+1}(t)$ vanishes identically in $(\alpha, \beta)$, then we have to consider solutions of (1.1) for which $y_{1}(t) \equiv y_{2}(t) \equiv \cdots \equiv y_{q}(t) \equiv 0$ in $(\alpha, \beta)$ and the remaining components satisfy a system of order $n-q$. Theorem 5 
does therefore not hold for TP systems; the following weakened version is however valid for such systems.

THEOREM 7. Let the differential system

$$
y^{\prime}(t)=A(t) y(t)
$$

be TP in $(a, b),-\infty \leqq a<b \leqq \infty$, and let $y(t)=\left(y_{1}(t), \cdots, y_{n}(t)\right)$ be $a$ nontrivial solution.

(i ) If $S^{+}(y(r))=0, r \in(a, b)$, then no component of $y(t)$ vanishes in $[r, b)$. If $S^{-}(y(s))=n-1, s \in(a, b)$, then no component of $y(t)$ vanishes in $(a, s]$.

(ii) Let

$$
(a<) \gamma_{0}<\alpha_{1}<\gamma_{1}<\alpha_{2}<\cdots<\gamma_{k-1}<\alpha_{k}<\gamma_{k}(<b)
$$

be $2 k+1$ points, such that for each $i, i=1, \cdots, k$, at least one of the following two conditions holds.

$$
y_{1}\left(\gamma_{i-1}\right) \neq 0, y_{1}\left(\alpha_{i}\right)=0, y_{1}\left(\gamma_{i}\right) \neq 0,
$$

or

$$
y_{n}\left(\gamma_{i-1}\right) \neq 0, y_{n}\left(\alpha_{i}\right)=0, y_{n}\left(\gamma_{i}\right) \neq 0 .
$$

Set $m_{i}=1$ if only one of these two conditions holds for the index $i$, and $m_{i}=2$ if both conditions hold, $i=1, \cdots, k$, and let $\tilde{k}=\sum_{i=1}^{k} m_{i}$. Then $\widetilde{k} \leqq n-1$. Moreover, if $\widetilde{k}=n-1$, then no component of $y(t)$ vanishes in $\left(a, \gamma_{0}\right] \cup\left[\gamma_{k}, b\right)$.

(iii) Let all the assumptions of (ii) hold and, in addition, assume that $m$ components of $y(r), r \in(a, b)$ vanish and that either $r \leqq \gamma_{0}$ or $\gamma_{k} \leqq r . \quad$ Then $\widetilde{k} \leqq n-m-1$.

Proof. ( i ) (5.1), (5.2) and (4.6) yield these two assertions. (ii) We have

$$
\begin{aligned}
\widetilde{k} \leqq & \sum_{i=1}^{k}\left[S^{+}\left(y\left(\alpha_{i}\right)\right)-S^{+}\left(y\left(\gamma_{i}\right)\right)\right]=\sum_{i=2}^{k}\left[S^{+}\left(y\left(\alpha_{i}\right)\right)-S^{+}\left(y\left(\gamma_{i-1}\right)\right)\right] \\
& +S^{+}\left(y\left(\alpha_{1}\right)\right)-S^{+}\left(y\left(\gamma_{k}\right)\right) \leqq S^{+}\left(y\left(\alpha_{1}\right)\right)-S^{+}\left(y\left(\gamma_{k}\right)\right) \leqq n-1 .
\end{aligned}
$$

The first inequality sign follows from (5.22) and (5.23) by Lemma 5 . The second inequality sign follows by (5.2). This proves $\tilde{k} \leqq n-1$. If $\widetilde{k}=n-1$, then (5.24) implies $S^{+}\left(y\left(\gamma_{k}\right)\right)=0$, hence part (i) implies that no component of $y(t)$ vanishes in $\left[\gamma_{k}, b\right)$. To show that, if $\widetilde{k}=$ $n-1$, no component vanishes in $\left(a, \gamma_{0}\right]$ either, we use

$$
\begin{aligned}
\tilde{k} \leqq & \sum_{i=1}^{k}\left[S^{-}\left(y\left(\gamma_{i-1}\right)\right)-S^{-}\left(y\left(\alpha_{i}\right)\right)\right]=\sum_{i=2}^{k}\left[S^{-}\left(y\left(\gamma_{i-1}\right)\right)-S^{-}\left(y\left(\alpha_{i-1}\right)\right)\right] \\
& +S^{-}\left(y\left(\gamma_{0}\right)\right)-S^{-}\left(y\left(\alpha_{k}\right)\right) \leqq S^{-}\left(y\left(\gamma_{0}\right)\right)-S^{-}\left(y\left(\alpha_{k}\right)\right) \leqq n-1 .
\end{aligned}
$$


(5.25) and $\widetilde{k}=n-1$ imply $S^{-}\left(y\left(\gamma_{0}\right)\right)=n-1$, which gives the desired nonvanishing in $\left(a, \gamma_{0}\right)$.

(iii) If $r \leqq \gamma_{0}$, then (5.25), (5.1) and (4.6) imply

$$
\widetilde{k} \leqq S^{-}\left(y\left(\gamma_{0}\right)\right)-S^{-}\left(y\left(\alpha_{k}\right)\right) \leqq S^{-}(y(r))-S^{-}\left(y\left(\alpha_{k}\right) \leqq n-m-1 .\right.
$$

If $\gamma_{k} \leqq r$, then (5.24), (5.2) and (4.6) give

$$
\widetilde{k} \leqq S^{+}\left(y\left(\alpha_{1}\right)\right)-S^{+}\left(y\left(\gamma_{k}\right)\right) \leqq S^{+}\left(y\left(\alpha_{1}\right)\right)-S^{+}(y(r)) \leqq n-m-1 .
$$

This completes the proof of Theorem 7 .

6. Examples. We conclude this paper with a few examples. All our examples are STP systems (1.1) and for each example we consider only one particular vector solution $y(t)$. We thus replace (1.1) in each case by a vector equality where the matrix $A(t)$, the particular solution $y(t)$ and its derivative $y^{\prime}(t)$ are shown explicitly. As the case $n=2$ is trivial, we start with an example for $n=3$.

$$
\left(\begin{array}{l}
-\sin t \\
-\cos t \\
-\sin t
\end{array}\right)=\left(\begin{array}{ccc}
0 & 1 & 0 \\
\frac{3}{2}-\cos t & 0 & \frac{3}{2}+\cos t \\
0 & 1 & 0
\end{array}\right)\left(\begin{array}{r}
2+\cos t \\
-\sin t \\
-2+\cos t
\end{array}\right) .
$$

This shows that, for $n=3$, there exists a system (1.1) which is STP in $(-\infty, \infty)$ and for which the interior component $y_{2}(t)$ of a particular solution $y(t)$ vanishes infinitely many times. However, in this example the extreme components $y_{1}(t)$ and $y_{3}(t)$ do not vanish at all.

The next examples show that the assertion of Theorem 5, is, for $n=3$, essentially all that can be said about the number of zeros of the components of any particular solution $y(t)$ of a STP system. Let $\alpha$ and $\beta, \alpha<\beta$, be zeros of the extreme components $y_{1}(t)$ and $y_{3}(t)$. Theorem 5 (ii) implies that these extreme components have no other zeros and that $y_{2}(t)$ does not vanish outside the interval $(\alpha, \beta)$; however, no restrictions on the number of zeros of $y_{2}(t)$ in $(\alpha, \beta)$ are given by Theorem 5. We combine system (6.1) with two other systems to show that we may obtain an (except for its parity) arbitrary number of zeros of $y_{2}(t)$ in the interval bounded by the zeros of the extreme components. The matrix in (6.1) and the vector given there, will be referred to as $A(t)$ and $y(t)$. We now consider the equality

$$
\left(\begin{array}{l}
1 \\
0 \\
1
\end{array}\right)=\left(\begin{array}{ccc}
0 & 1 & 0 \\
\frac{3}{4}(2-\tau) & 0 & \frac{3}{4}(2+\tau) \\
0 & 1 & 0
\end{array}\right)\left(\begin{array}{c}
2+\tau \\
2 \\
-2+\tau
\end{array}\right) .
$$

Note that the corresponding system 


$$
u^{\prime}(\tau)=B(\tau) u(\tau),
$$

is STP in [-2,2]. Furthermore we note that if now $u(\tau)$ denotes the particular solution shown in (6.2), then, for each integer $k$, the equalities

$$
B(0)=A\left(-\frac{\pi}{2}+2 k \pi\right), u(0)=y\left(-\frac{\pi}{2}+2 k \pi\right),
$$

(and hence also $u^{\prime}(0)=y^{\prime}(-\pi / 2+2 k \pi)$ hold. (6.3) allows us to combine the examples (6.1) and (6.2) at their respective points $t=$ $-\pi / 2+2 k \pi$ and $\tau=0$.

We also consider the equality

$$
\left(\begin{array}{r}
-1 \\
0 \\
-1
\end{array}\right)=\left(\begin{array}{ccc}
0 & 1 & 0 \\
\frac{3}{4}(2+\tau) & 0 & \frac{3}{4}(2-\tau) \\
0 & 1 & 0
\end{array}\right)\left(\begin{array}{c}
2-\tau \\
-1 \\
-2-\tau
\end{array}\right) .
$$

The corresponding system

$$
v^{\prime}(\tau)=C(\tau) v(\tau)
$$

is again STP in $[-2,2]$ and, for each integer $k$,

$$
C(0)=A\left(\frac{\pi}{2}+2 k \pi\right), v(0)=y\left(\frac{\pi}{2}+2 k \pi\right),
$$

and we thus may combine (6.1) and (6.4).

For any nonnegative integer $k$, we now define the system $(6.6)_{1}$

$$
\left[y^{(1)}(t)\right]^{\prime}=A^{(1)}(t) y^{(1)}(t),
$$

in $\left[\alpha_{1}, \beta_{1}\right], \alpha_{1}=-2-\pi / 2, \beta_{1}=\pi / 2+2 k \pi+2$, by setting

$$
A^{(1)}(t)=\left\{\begin{array}{lr}
B\left(t+\frac{\pi}{2}\right), & -2-\frac{\pi}{2} \leqq t \leqq-\frac{\pi}{2}, \\
A(t), & -\frac{\pi}{2} \leqq t \leqq \frac{\pi}{2}+2 k \pi, \\
C\left(t-\frac{\pi}{2}-2 k \pi\right), & \frac{\pi}{2}+2 k \pi \leqq t \leqq \frac{\pi}{2}+2 k \pi+2 .
\end{array}\right.
$$

This systems is STP in $\left[\alpha_{1}, \beta_{1}\right]$ and has the particular solution

$$
y^{(1)}(t)=\left\{\begin{array}{lr}
u\left(t+\frac{\pi}{2}\right), & -2-\frac{\pi}{2} \leqq t \leqq \frac{\pi}{2}, \\
y(t), & -\frac{\pi}{2} \leqq t \leqq \frac{\pi}{2}+2 k \pi, \\
v\left(t-\frac{\pi}{2}-2 k \pi\right), & \frac{\pi}{2}+2 k \pi \leqq t \leqq \frac{\pi}{2}+2 k \pi+2 .
\end{array}\right.
$$


$y_{1}^{(1)}\left(\alpha_{1}\right)=y_{1}^{(1)}\left(\beta_{1}\right)=0, y_{3}^{(1)}(t)<0$ in $\left[\alpha_{1}, \beta_{1}\right]$, and $y_{2}^{(1)}(t)$ vanishes at the $2 k+1$ points $t=\ell \pi, \iota=0,1, \cdots, 2 k$.

If we define the system

$$
\left[y^{(2)}(t)\right]^{\prime}=A^{(2)}(t) y^{(2)}(t),
$$

in $\left[\alpha_{2}, \beta_{2}\right], \alpha_{2}=-2+\pi / 2, \beta_{2}=3 \pi / 2+2 k \pi+2,(k=0,1, \cdots)$, by setting

$$
A^{(2)}(t)=\left\{\begin{array}{lr}
C\left(t-\frac{\pi}{2}\right), & -2+\frac{\pi}{2} \leqq t \leqq \frac{\pi}{2}, \\
A(t), & \frac{\pi}{2} \leqq t \leqq \frac{3 \pi}{2}+2 k \pi, \\
B\left(t-\frac{3 \pi}{2}-2 k \pi\right), & \frac{3 \pi}{2}+2 k \pi \leqq t \leqq \frac{3 \pi}{2}+2 k \pi+2,
\end{array}\right.
$$

then this system has a solution $y^{(2)}(t)$, for which $y_{3}^{(2)}\left(\alpha_{2}\right)=y_{3}^{(2)}\left(\beta_{2}\right)=0$, $y_{1}^{(2)}(t)>0$ in $\left[\alpha_{2}, \beta_{2}\right]$, and $y_{2}^{(2)}(t)$ has again an odd number of zeros in $\left[\alpha_{2}, \beta_{2}\right]$.

Defining $A^{(3)}(t)$ in $\left[\alpha_{3}, \beta_{3}\right], \alpha_{3}=-2-\pi / 2, \beta_{3}=3 \pi / 2+2 k \pi+2$, by

$$
A^{(3)}(t)=\left\{\begin{array}{lr}
B\left(t+\frac{\pi}{2}\right), & -2-\frac{\pi}{2} \leqq t \leqq \frac{\pi}{2}, \\
A(t), & -\frac{\pi}{2} \leqq t \leqq \frac{3 \pi}{2}+2 k \pi, \\
B\left(t-\frac{3 \pi}{2}-2 k \pi\right), & \frac{3 \pi}{2}+2 k \pi \leqq t \leqq \frac{3 \pi}{2}+2 k \pi+2,
\end{array}\right.
$$

we obtain a system which is $\mathrm{STP}$ in $\left[\alpha_{3}, \beta_{3}\right]$ and has a solution $y^{(3)}(t)$, for which $y_{1}^{(3)}\left(\alpha_{3}\right)=y_{3}^{(3)}\left(\beta_{3}\right)=0$, and $y_{2}^{(3)}(t)$ has now an even number of zeros in $\left[\alpha_{3}, \beta_{3}\right]$. By using first $C$, then $A$ and then again $C$, we obtain similarly a fourth example for which $y_{3}^{(4)}\left(\alpha_{4}\right)=y_{1}^{(4)}\left(\beta_{4}\right)=0$ and $y_{2}^{(4)}(t)$ has again an even number of zeros in $\left[\alpha_{4}, \beta_{4}\right]$. These four examples establish the italicized statement preceding (6.2); the parity restriction on the number of the zeros of $y_{2}(t)$ follows easily by the proof of part (ii) of Theorem 5. We remark that there is no need to consider different systems for all four examples and all nonnegative integers $k$. All these cases can be illustrated by considering distinct solutions $y^{(j, k)}, j=1, \cdots, 4, k=0,1, \cdots$, of a single system (1.1) which is STP in $(-\infty, \infty)$. The corresponding matrix $A(t)$ is given by $a_{11}(t)=a_{13}(t)=a_{31}(t)=a_{33}(t)=0$ and $a_{12}(t)=a_{32}(t)=1$ for all $t$, while the elements $a_{21}(t)$ and $a_{23}(t)$ are determined in the disjoint intervals $\left[\alpha_{j k}, \beta_{j k}\right]$ by the above formulas and are (otherwise arbitrary) continuous nonnegative functions of $t$, which only vanish at some of the end points $\alpha_{j k}$ and $\beta_{j k}$.

For $n=4$ our example is 


$$
\left(\begin{array}{l}
\sin t \\
\cos t \\
\cos t \\
\sin t
\end{array}\right)=\left(\begin{array}{cccc}
\frac{-1}{3(2-\cos t)} & 1 & 0 & 0 \\
1+\cos t & -\frac{5}{3} & \frac{4}{3}-\sin t & 0 \\
0 & \frac{4}{3}+\sin t & -\frac{5}{3} & 1-\cos t \\
0 & 0 & 1 & \frac{-1}{3(2+\cos t)}
\end{array}\right)
$$

$$
\times\left(\begin{array}{r}
2-\cos t \\
\frac{1}{3}+\sin t \\
-\frac{1}{3}+\sin t \\
-2-\cos t
\end{array}\right)
$$

The corresponding system is STP in $(-\infty, \infty)$ and the interior components $y_{2}(t)$ and $y_{3}(t)$ of the particular solution $y(t)$ have infinitely many zeros. (6.7) is the special case $n=4$ of a general example, valid for any $n, n \geqq 4$. The nonzero elements of the Jacobi matrix $A(t)=\left(a_{i j}(t)\right)_{1}^{n}$ are in this general case given by

$$
\begin{aligned}
a_{11} & =\frac{3-n}{(n-1)(2-\cos t)}, a_{12}=1, a_{21}=1+\cos t, \\
a_{22} & =5-n-\frac{8}{n-1}, a_{23}=n-4+\frac{4}{n-1}-\sin t, \\
a_{i i-1} & =\frac{n-1}{2}\left(1+\frac{1}{2} \cos t\right), a_{i i}=1-n, \\
a_{i i+1} & =\frac{n-1}{2}\left(1-\frac{1}{2} \cos t\right), i=3, \cdots, n-2, \\
a_{n-1, n-2} & =n-4+\frac{4}{n-1}+\sin t, a_{n-1, n-1}=5-n-\frac{8}{n-1}, \\
a_{n-1, n} & =1-\cos t, a_{n n-1}=1, a_{n n}=\frac{3-n}{(n-1)(2+\cos t)} .
\end{aligned}
$$

The particular solution is the vector $y(t)$, whose components are

$$
\begin{aligned}
& y_{1}(t)=2-\cos t, \\
& y_{i}(t)=\frac{n+1-2 i}{n-1}+\sin t, \quad i=2, \cdots, n-1, \\
& y_{n}(t)=-2-\cos t,
\end{aligned}
$$


and all interior components vanish infinitely many times.

I am grateful to Professor Z. Nehari and Dr. M. Lavie for their valuable advice offered during many discussions.

\section{REFERENCES}

1. R. Bellman, Introduction to matrix analysis, McGraw-Hill, New York, 1960.

2. F. R. Gantmacher and M. G. Krein, Oszillationsmatrizen, Oszillationskerne und kleine Schwingungen mechanischer Systeme, Akademie-Verlag, Berlin, 1960.

3. P. Hartman, Ordinary differential equations, John Wiley and Sons, New York, 1964.

4. S. Karlin, Total positivity, vol. 1, Stanford University press, Stanford, 1968.

5. C. Loewner, On totally positive matrices, Math. Z. 63 (1955), 338-340.

6. J. Mikusiński, Sur l'equation $x^{(n)}+A(t) x=0$, Ann. Polon. Math. 1 (1955), 207-221.

7. Z. Nehari, Disconjugate linear differential operators, Trans. Amer. Math. Soc. 129 (1967), 500-516.

8. I. Schoenberg, Uber variationsvermindernde lineare Transformationen, Math. Z. 32 (1930), 321-328.

9. R. S. Varga, Matrix iterative analysis. Prentice-Hall, Englewood Cliffs, New Jersey, 1962.

Received June 5, 1969.

Carnegie-Mellon University, Pittsburgh, Pennsylvania

TeChNiON-ISRAEL INSTitute OF Technology, HAIFA, ISRAEL 



\section{PACIFIC JOURNAL OF MATHEMATICS}

\section{EDITORS}

\author{
H. SAMELSON \\ Stanford University \\ Stanford, California 94305 \\ Richard Pierce \\ University of Washington \\ Seattle, Washington 98105
}

J. DUGUNDJI

Department of Mathematics

University of Southern California

Los Angeles, California 90007

BASIL GORDON*

University of California

Los Angeles, California 90024

\section{ASSOCIATE EDITORS}
E. F. BECKENBACH
B. H. NeUmanN
F. WOLF
K. YoSHIDA

\section{SUPPORTING INSTITUTIONS}

UNIVERSITY OF BRITISH COLUMBIA CALIFORNIA INSTITUTE OF TECHNOLOGY

UNIVERSITY OF CALIFORNIA MONTANA STATE UNIVERSITY

UNIVERSITY OF NEVADA

NEW MEXICO STATE UNIVERSITY

OREGON STATE UNIVERSITY

UNIVERSITY OF OREGON

OSAKA UNIVERSITY

UNIVERSITY OF SOUTHERN CALIFORNIA
STANFORD UNIVERSITY

UNIVERSITY OF TOKYO

UNIVERSITY OF UTAH

WASHINGTON STATE UNIVERSITY

UNIVERSITY OF WASHINGTON

AMERICAN MATHEMATICAL SOCIETY CHEVRON RESEARCH CORPORATION TRW SYSTEMS

NAVAL WEAPONS CENTER 


\section{Pacific Journal of Mathematics}

\section{Vol. 32, No. $1 \quad$ January, 1970}

Robert Alexander Adams, Compact Sobolev imbeddings for unbounded domains ........................................ 1

Bernhard Amberg, Groups with maximum conditions .................. 9

Tom M. (Mike) Apostol, Möbius functions of order k............... 21

Stefan Bergman, On an initial value problem in the theory of two-dimensional transonic flow patterns ................... 29

Geoffrey David Downs Creede, Concerning semi-stratifiable spaces ...... 47

Edmond Dale Dixon, Matric polynomials which are higher

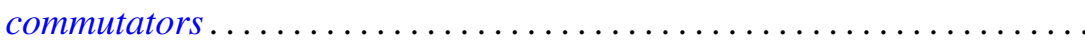

R. L. Duncan, Some continuity properties of the Schnirelmann density.

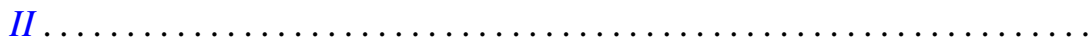

Peter Larkin Duren and Allen Lowell Shields, Coefficient multipliers of $H^{p}$

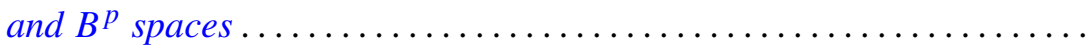

Hector O. Fattorini, On a class of differential equations for vector-valued

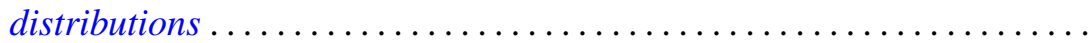

Charles Hallahan, Stability theorems for Lie algebras of derivations. . . . . . 105

Heinz Helfenstein, Local isometries of flat tori ................ 113

Gerald J. Janusz, Some remarks on Clifford's theorem and the Schur

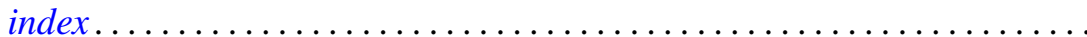

Joe W. Jenkins, Symmetry and nonsymmetry in the group algebras of discrete groups. ...

Herbert Frederick Kreimer, Jr., Outer Galois theory for separable

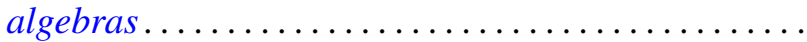

D. G. Larman and P. Mani, On visual hulls

R. Robert Laxton, On groups of linear recurrences. II. Elements of finite order.

Dong Hoon Lee, The adjoint group of Lie groups ...

James B. Lucke, Commutativity in locally compact rings

Charles Harris Scanlon, Rings of functions with certain Lipschitz

$$
\text { properties ............................... }
$$

Binyamin Schwarz, Totally positive differential systems .

James McLean Sloss, The bending of space curves into piecewise helical curves.

James D. Stafney, Analytic interpolation of certain multiplier spaces ...

Patrick Noble Stewart, Semi-simple radical classes.......

Hiroyuki Tachikawa, On left $\mathrm{QF}-3$ rings ...................... 255

Glenn Francis Webb, Product integral representation of time dependent nonlinear evolution equations in Banach spaces.... . . . 